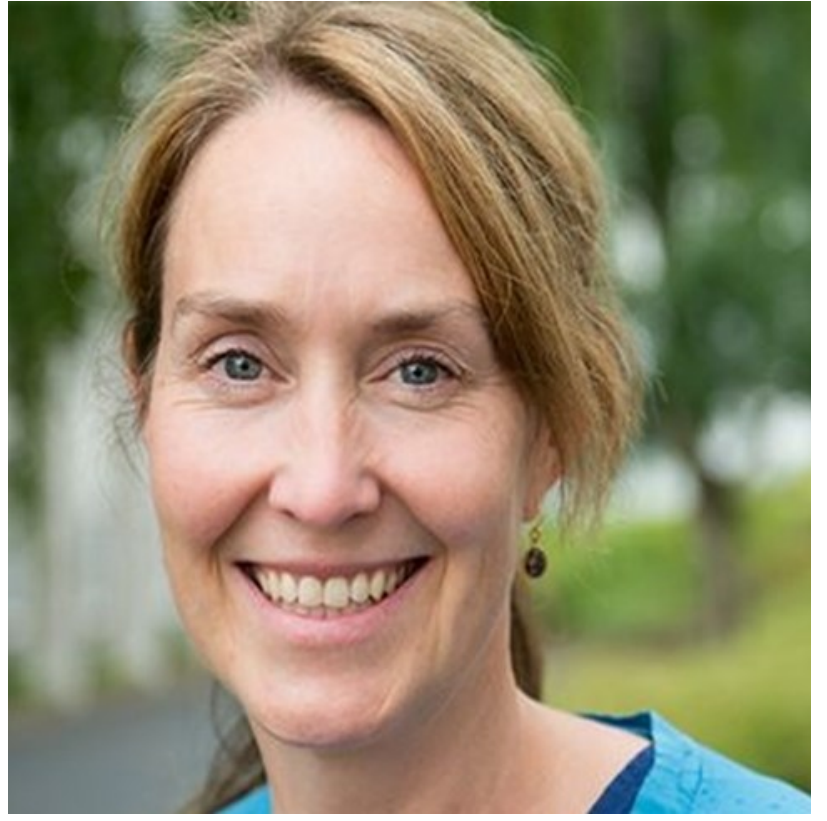

Charlotte Runge, MD, PhD

Centre of Elective Surgery

Silkeborg Regional Hospital

Denmark

Email: charlotte.runge@aarhus.rm.dk

\title{
An Old Approach to a New Blockade - the Effect of Popliteal Plexus Blockade after Total Knee Arthroplasty
}

Runge C, PhD, Bjørn S, MS, Jensen JM, MD, Nielsen ND, MD, Vase M, Holm C, MD, Bendtsen TF, MD, PhD

\section{A feasibility study}

\section{Introduction}

Total knee arthroplasty (TKA) is associated with:

- $\quad$ severe pain

- $\quad$ high postoperative opioid consumption

- importance of early and effective rehabilitation

\section{Background}

- A combined femoral triangle and obturator nerve block effectively reduces pain and opioid comsumption after TKA

- Popliteal plexus is formed by contributions from the obturator and the tibial nerve.

- In cadavers injection of $10 \mathrm{~mL}$ of dye in the distal adductor canal spreads to the popliteal fossa and stains the popliteal plexus and the posterior obturator nerve

\section{Femoral triangle block}
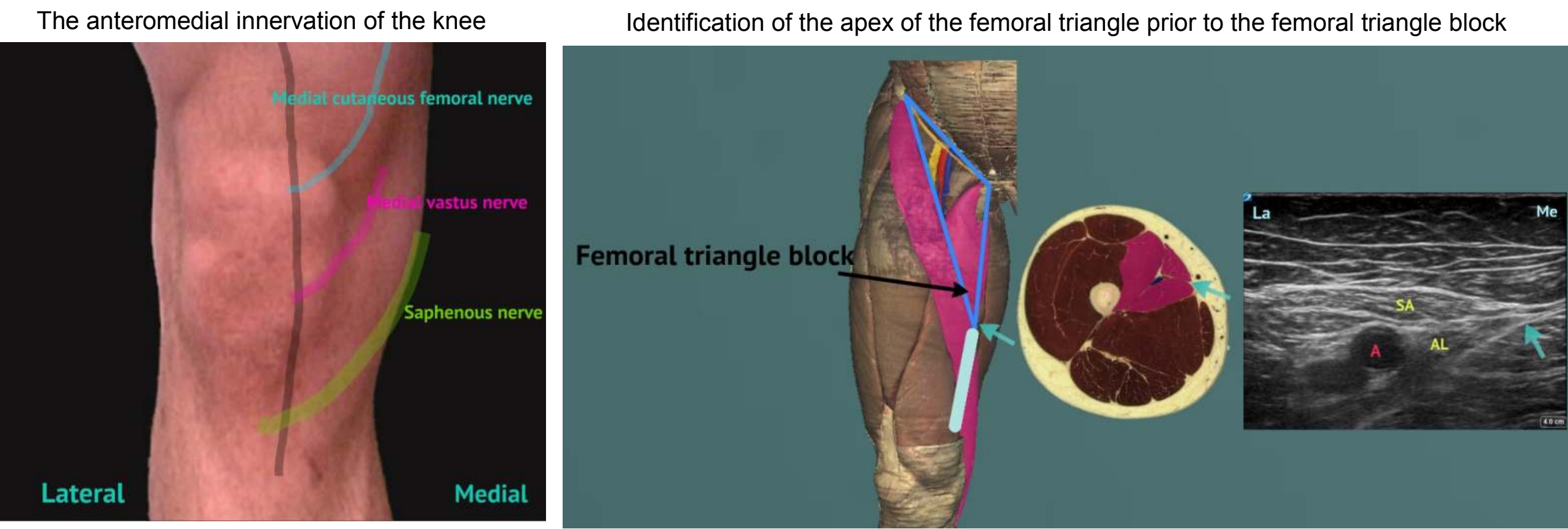

Endpoint of injection by a femoral triangle

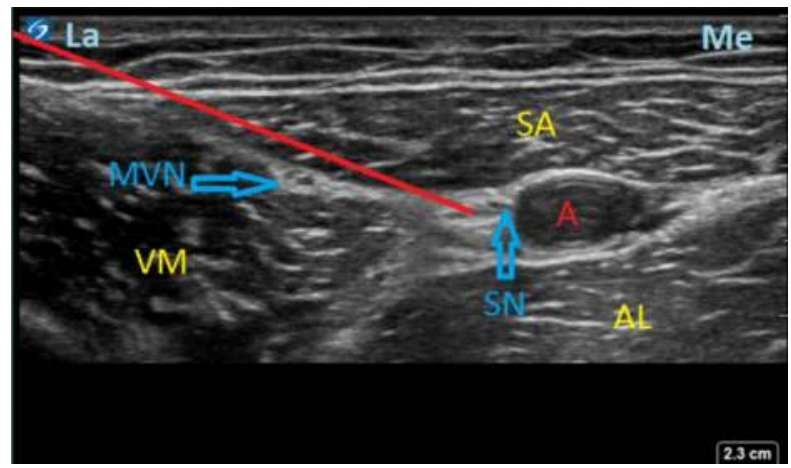

$\mathrm{La}=$ lateral, $\mathrm{Me}=$ medial, $\mathrm{A}=$ femoral artery, $\mathrm{SA}=$ sartorius muscle, $\mathrm{AL}=$ adductor longus muscle, $\mathrm{MVN}=$ medialis vastus nerve, $\mathrm{SN}=$ saphenous nerve, $\mathrm{VM}=$ vastus medialis muscle

\section{Popliteal plexus block}

The posterlou knectinervation

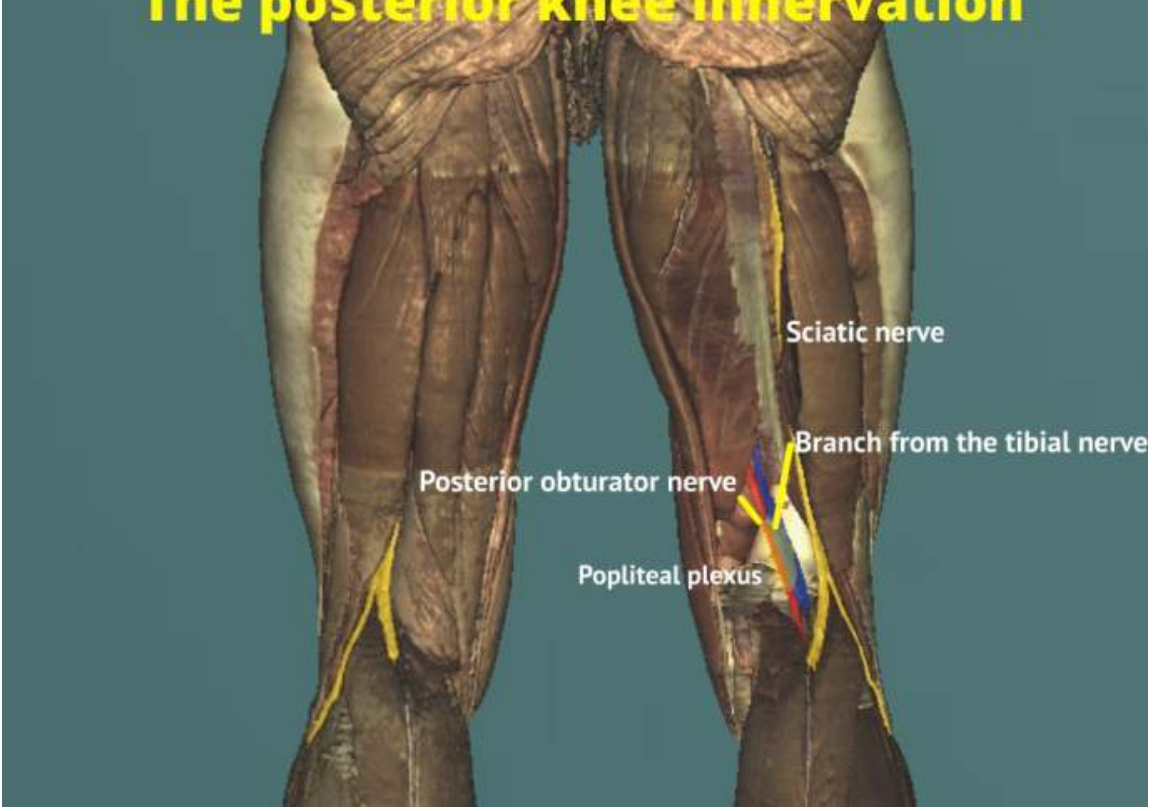

\section{Methods}

- $\quad$ femoral triangle block was performed preoperatively

- $\quad$ spinal anesthesia for the surgery

- $\quad$ if postoperative pain $>3$

- $\quad$ then a popliteal plexus block was performed

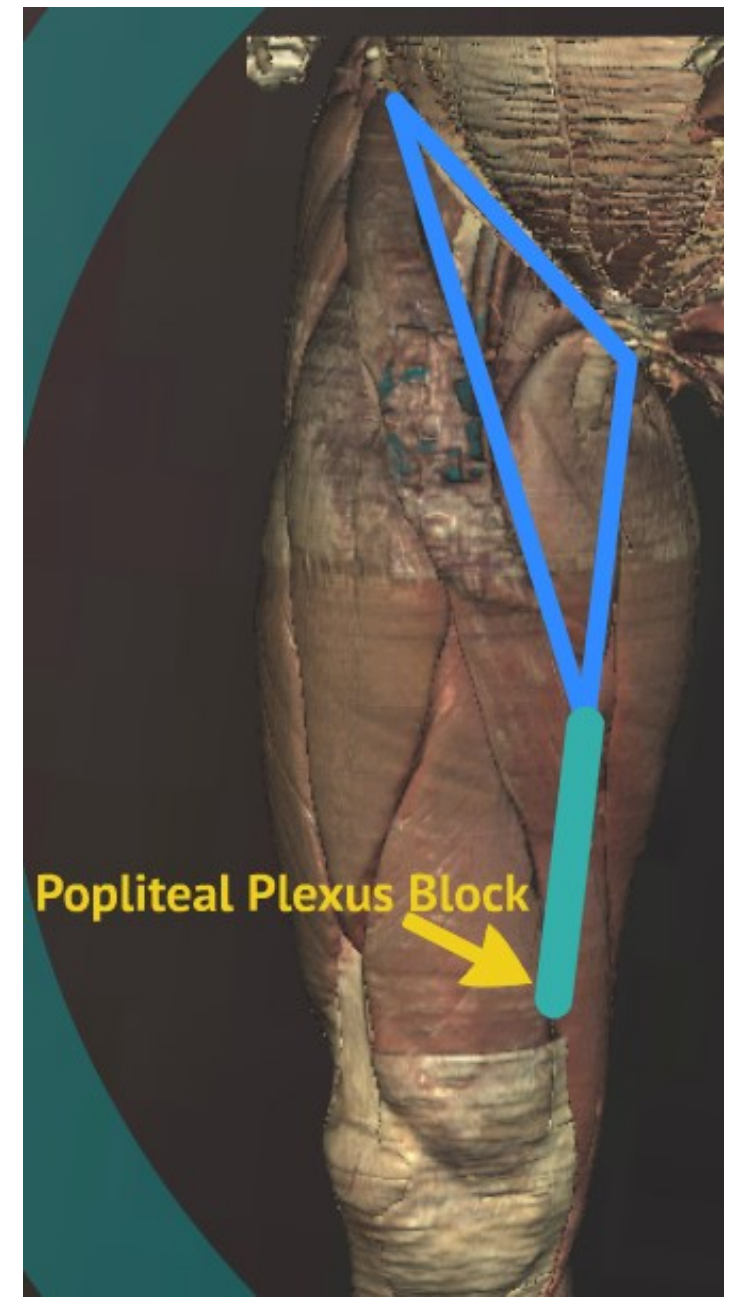

Endpoint of injection by a popliteal plexus block

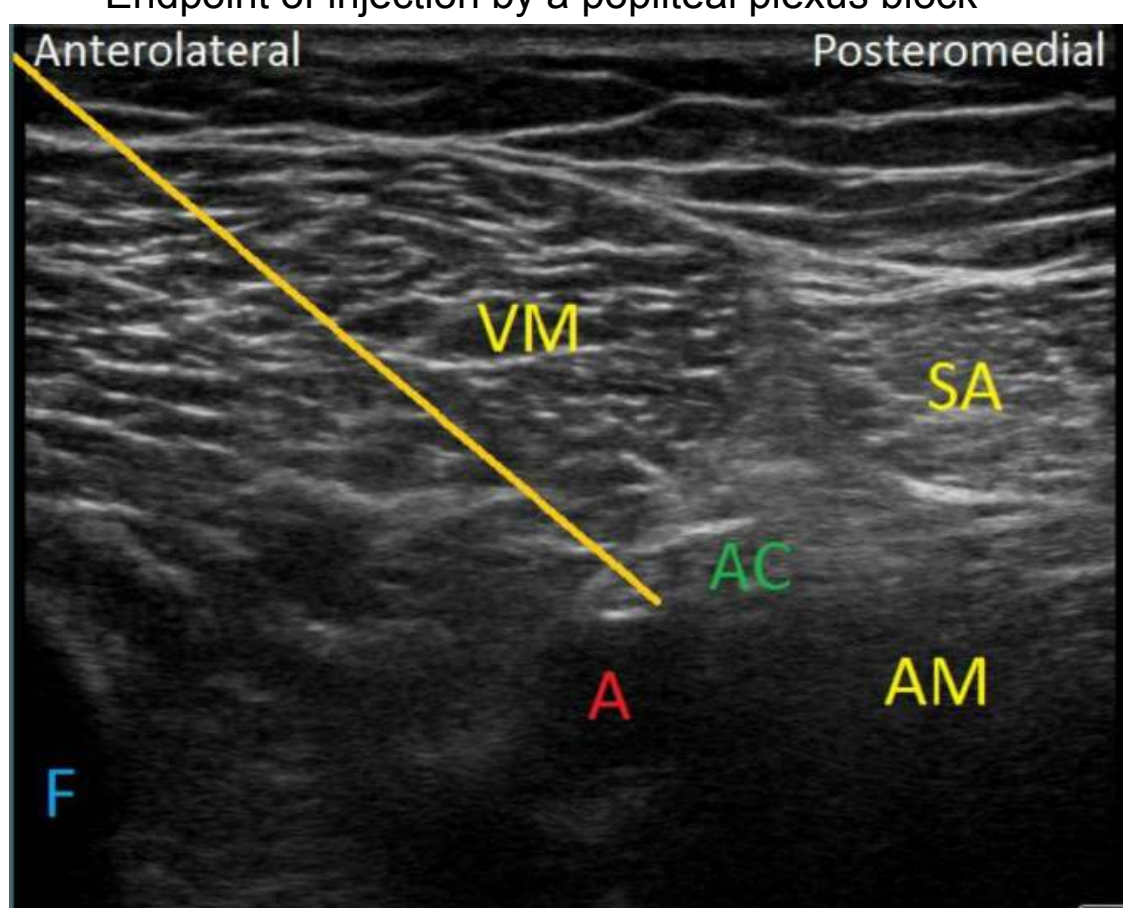

$\mathrm{VM}=$ vastus medialis muscle, $\mathrm{SA}=$ sartorius muscle, $\mathrm{A}=$ femoral artery, $A C=$ adductor canal, $A M=$ adductor magnus muscle, $F=$ femoral bone

\section{Results}

In 10 Patients:

- $\quad$ NRS: 5.5 (4-8) prior to Popliteal Plexus Block (PPB)

- $\quad \mathrm{NRS}<3$ within 10 minutes after $\mathrm{PPB}$

- $\quad$ no motor block of the motor function of the sciatic nerve

\section{Conclusion}

Popliteal plexus block appears to have a supplemental effect on pain after total knee arthroplasty without affecting the motor function. 